\title{
Biased geodetic inference on asperity distribution on a subducted plate interface: a quantitative study
}

\author{
Takashi Hirai $^{1}$ and Takeshi Sagiya ${ }^{2}$ \\ ${ }^{1}$ Graduate School of Environmental Studies, Nagoya University, Furocho, Chikusa-ku, Nagoya 464-8601, Japan \\ ${ }^{2}$ Disaster Mitigation Research Center, Nagoya University, Furocho, Chikusa-ku, Nagoya 464-8601, Japan
}

(Received May 1, 2012; Revised July 26, 2012; Accepted August 22, 2012; Online published May 7, 2013)

\begin{abstract}
The asperity model was developed to explain plate boundary behavior such as interplate earthquakes. Asperity is defined as a strongly-coupled region on the plate interface. Since interplate earthquakes are considered to occur on asperities, it is important to know the asperity distribution, which can be inferred from interseismic crustal deformation through estimation of the slip deficit distribution. Slip deficit is the difference between the long-term plate convergence rate and the actual relative displacement rate of the plate interface. It is a kinematic description of plate interaction. The relation between the estimated slip deficit and the asperity is still not clearly understood. We have conducted a quantitative comparison between them by combining a forward simulation of crustal deformation, as a result of plate subduction, and a geodetic data inversion. We found that the seismic moment accumulation rate is likely to be overestimated in most cases. The degree of overestimation increases in the case of small asperity areas. Conversely, if no slip deficit is detected by geodetic data inversion, it is highly probable that no asperity exists. Such a misinference may lead to an incorrect estimation of strong ground motion in future earthquakes, and appropriate measures should be taken to allow for this.
\end{abstract}

Key words: Asperity, crustal deformation, geodetic data inversion, interplate earthquake, slip deficit.

\section{Introduction}

In plate subduction zones, large interplate earthquakes occur repeatedly at the interface between subducting oceanic, and overriding continental, plates. An interplate earthquake occurs when the shear stress on the plate interface caused by plate subduction reaches its frictional strength.

Through detailed analyses of seismic waves, we observed that coseismic slip distribution is not uniform in the source area. Based on such an observation, Lay and Kanamori (1981) proposed the asperity model. They called the region in which a large fault slip occurs an "asperity," and assumed that seismic energy is released mainly in these asperities.

Around the Japan arc, many large interplate earthquakes have struck along the Japan Trench and the Suruga-Nankai Trough, occurring every several decades. Nagai et al. (2001) found that the 1968 Tokachi-Oki earthquake and the 1994 Sanriku-Haruka-Oki earthquake had a large fault slip in the same region of the plate interface. Recently, Moreno et al. (2010, 2012) determined that the Maule (Chile) earthquake of February 27, 2010, ruptured a seismic gap of the Andean subduction zone megathrust. These results suggest that asperities are persistent through time and that the asperity distribution represents the spatial heterogeneity of frictional strength on the plate interface. Thus, a knowledge of the spatial distribution of asperities on the plate in-

Copyright (C) The Society of Geomagnetism and Earth, Planetary and Space Sciences (SGEPSS); The Seismological Society of Japan; The Volcanological Society of Japan; The Geodetic Society of Japan; The Japanese Society for Planetary Sciences; TERRAPUB.

doi:10.5047/eps.2012.08.008 terface is fundamental information for studying interplate earthquakes.

Asperity distribution on the plate interface can be inferred in various ways. Seismologically, asperities are identified as large slip patches on the source fault inferred from the analysis of seismic waveforms (e.g., Kikuchi et al., 2003). This is a standard method for estimating asperity distribution, but it is not applicable to cases where no seismological records exist. Conversely, geodetic investigations can provide independent information about asperities. For the transform fault, Harris and Segall (1987) inverted the geodetic observation data around the San Andreas fault to determine the buried slip on the fault. For the subducting plate interface, Yoshioka et al. (1993) developed a geodetic data inversion method that uses the interseismic crustal deformation rate to estimate the interplate coupling distribution. The interplate coupling is represented by slip deficit, which is the difference between the long-term relative displacement rate on the plate interface, reflecting the plate motion, and the actual relative displacement rate, reflecting interplate locking. Slip deficit inversion has been widely applied to precise geodetic data obtained by GPS measurements (e.g., Ito et al., 1999; Sagiya, 1999; Ohta et al., 2004). However, it should be noted that slip deficit distribution is a kinematic description of the plate boundary process, unlike asperity distribution that reflects the mechanical heterogeneity on the plate interface. A quantitative comparison between slip deficit and asperities has not been conducted so far.

In this study, we evaluate the relation between the asperity distribution and the slip deficit distribution in a quan- 
titative manner. For this purpose, we calculate the theoretical slip deficit distribution for a given asperity distribution. Then, we synthesize surface displacement data and analyze them to estimate the slip deficit distribution. Next, we conduct a quantitative comparison between the assumed asperity distribution and the estimated slip deficit distribution; hence, we can determine the difference in size and position on the plate interface between the true asperity and the estimated slip deficit. Asperity size is important information for predicting the magnitude of future earthquakes. The position of the asperity affects the strong ground motion prediction, since seismic waves attenuate rapidly with the distance between the observation site and the asperity.

In Section 2, we formulate the problem. Then, we examine several numerical examples in Section 3, and discuss the results in Section 4, followed by conclusions in Section 5.

\section{Method for Numerical Simulation and Analysis 2.1 Forward simulation}

According to Savage (1983), the down-dip component of the relative displacement rate on the plate interface $\Delta w(\boldsymbol{x}, t)$ is represented as the sum of the steady relative plate convergence rate $v_{p l}$ and its perturbation term $\Delta w_{s}(\boldsymbol{x}, t)$ :

$$
\Delta w(\boldsymbol{x}, t)=v_{p l}+\Delta w_{s}(\boldsymbol{x}, t)
$$

Here, $\boldsymbol{x}$ denotes an arbitrary point on the plate interface and $t$ denotes the time with an appropriate reference. Only the down-dip component is considered in our study. We call $\Delta w_{s}(\boldsymbol{x}, t)$ the slip deficit rate hereafter. The slip deficit rate changes in both space and time, reflecting the coupling status on the plate interface. During the interseismic time period, the plate interface is locked in, and around, the asperities, and the relative displacement between the subducting oceanic plate and the overriding continental plate does not increase in time, causing negative $\Delta w_{s}(\boldsymbol{x}, t)$. The shear stress accumulation rate arising from the interplate slip is expressed as:

$$
\sigma(\boldsymbol{x}, t)=\int_{S} H(\boldsymbol{x}, t ; \boldsymbol{\xi}, \tau) \Delta w(\boldsymbol{\xi}, \tau) d S(\boldsymbol{\xi}) .
$$

Here, $H(\boldsymbol{x}, t ; \boldsymbol{\xi}, \tau)$ denotes the stress response function representing the shear stress at point $\boldsymbol{x}$ because of a unit slip at point $\boldsymbol{\xi}$. The spatial integral on the right-hand side is applied over the whole plate interface. The steady plate motion determines the basic stress level. Conversely, slip deficit causes stress perturbation from the basic level. Thus, the stress change rate owing to plate motion is expressed as:

$$
\Delta \sigma(\boldsymbol{x}, t)=\int_{S} H(\boldsymbol{x} ; \boldsymbol{\xi}) \Delta w_{s}(\boldsymbol{\xi}, t) d S(\boldsymbol{\xi}) .
$$

The derivation of $H(\boldsymbol{x} ; \boldsymbol{\xi})$ is given in the Appendix. A fault slip on the plate interface occurs satisfying the constitutive relation between the fault slip and shear stress. In general, the constitutive relation is expressed as follows:

$$
\Delta \sigma(\boldsymbol{x}, t)=f[\Delta w(\boldsymbol{x}, t) ; \boldsymbol{x}] .
$$

Combining Eqs. (1), (3), and (4), the slip deficit and shear stress distribution on the plate interface can be calculated numerically.
Since we are dealing with interseismic crustal deformation in this study, we assume that the slip rate is zero inside the asperity and that the shear stress does not accumulate outside the asperity. Therefore, the problem is reduced by solving the coupled equation system (1) and (3) with the following constraints:

$$
\begin{array}{ll}
\Delta w(\boldsymbol{x}, t)=0 & \text { (inside an asperity) } \\
\Delta \sigma(\boldsymbol{x}, t)=0 & \text { (outside an asperity) }
\end{array}
$$

and Eqs. (5) and (6) are the most simple formulations of frictional heterogeneity of the plate interface. To consider the frictional property of the plate interface more carefully, the slip weakening friction law (Carlson et al., 1991), or the rate and state dependent friction law (Dieterich, 1979; Ruina, 1983), can be brought to bear. However, these friction laws have many parameters in their formulation, causing a complication of the problem. Since the relation between the mechanical and the kinematic interplate coupling is discussed in this study, we assume Eqs. (5) and (6) as the frictional constitutive relation of the plate interface for simplicity. The conclusion may not be significantly affected by a choice of friction model.

In order to solve the coupled equation system (1) and (3) numerically, we discretize these equations in space. We divide the model plate interface into $N$ small subfaults:

$$
\begin{aligned}
& \Delta w\left(\boldsymbol{x}_{i}, t\right)=v_{p l}+\Delta w_{s}\left(\boldsymbol{x}_{i}, t\right), \\
& \Delta \sigma\left(\boldsymbol{x}_{i}, t\right)=\sum_{j=1}^{N} H_{j}\left(\boldsymbol{x}_{i}\right) \Delta w_{s}\left(\boldsymbol{x}_{j}, t\right),
\end{aligned}
$$

where $H_{j}\left(\boldsymbol{x}_{i}\right)$ is the integral of the stress response function for subfault $j$,

$$
H_{j}\left(\boldsymbol{x}_{i}\right)=\int_{\Delta S_{j}} H\left(\boldsymbol{x}_{i}, \boldsymbol{\xi}\right) d S(\boldsymbol{\xi}) .
$$

These equations are written in matrix form as:

$$
\begin{aligned}
\Delta \boldsymbol{w} & =v_{p l} \mathbf{1}+\Delta \boldsymbol{w}_{s}, \\
\Delta \boldsymbol{\sigma} & =\boldsymbol{H} \Delta \boldsymbol{w}_{s},
\end{aligned}
$$

with

$$
\begin{aligned}
& \Delta \boldsymbol{w}^{T}=\left[\Delta w\left(\boldsymbol{x}_{1}, t\right) \Delta w\left(\boldsymbol{x}_{2}, t\right) \cdots \Delta w\left(\boldsymbol{x}_{N}, t\right)\right], \\
& \Delta \boldsymbol{w}_{s}^{T}=\left[\Delta w_{s}\left(\boldsymbol{x}_{1}, t\right) \Delta w_{s}\left(\boldsymbol{x}_{2}, t\right) \cdots \Delta w_{s}\left(\boldsymbol{x}_{N}, t\right)\right], \\
& \mathbf{1}^{T}=\left[\begin{array}{llll}
1 & 1 & \cdots & 1
\end{array}\right] \\
& \Delta \boldsymbol{\sigma}^{T}=\left[\Delta \sigma\left(\boldsymbol{x}_{1}, t\right) \Delta \sigma\left(\boldsymbol{x}_{2}, t\right) \cdots \Delta \sigma\left(\boldsymbol{x}_{N}, t\right)\right], \\
& \boldsymbol{H}=\left[\begin{array}{ccc}
H_{1}\left(\boldsymbol{x}_{1}\right) & \cdots & H_{N}\left(\boldsymbol{x}_{1}\right) \\
\vdots & \ddots & \vdots \\
H_{1}\left(\boldsymbol{x}_{N}\right) & \cdots & H_{N}\left(\boldsymbol{x}_{N}\right)
\end{array}\right] \text {. }
\end{aligned}
$$

Dividing the fault elements into the asperity (subscripted with " $a$ ") and non-asperity (subscripted with " $b$ "), Eqs. (10) and (11) are combined as:

$$
\left(\begin{array}{c}
\Delta \boldsymbol{\sigma}_{a} \\
\Delta \boldsymbol{\sigma}_{b}
\end{array}\right)=\left(\begin{array}{ll}
\boldsymbol{H}_{a a} & \boldsymbol{H}_{a b} \\
\boldsymbol{H}_{b a} & \boldsymbol{H}_{b b}
\end{array}\right)\left(\begin{array}{c}
\Delta \boldsymbol{w}_{a}-v_{p l} \mathbf{1}_{a} \\
\Delta \boldsymbol{w}_{b}-v_{p l} \mathbf{1}_{b}
\end{array}\right) .
$$


Here, $\Delta \boldsymbol{w}_{a}=\mathbf{0}$ and $\Delta \boldsymbol{\sigma}_{b}=\mathbf{0}$ refer to the constraints expressed by Eqs. (5) and (6). The solution of the slip distribution for a given plate velocity $v_{p l}$ can be calculated as:

$$
\Delta \boldsymbol{w}_{b}=v_{p l} \boldsymbol{H}_{b b}^{-1}\left(\boldsymbol{H}_{b a} \mathbf{1}_{a}+\boldsymbol{H}_{b b} \mathbf{1}_{b}\right)
$$

and

$$
\Delta \boldsymbol{\sigma}_{a}=v_{p l}\left(\boldsymbol{H}_{a b} \boldsymbol{H}_{b b}^{-1} \boldsymbol{H}_{b a}-\boldsymbol{H}_{a a}\right) \mathbf{1}_{a} .
$$

Once the slip deficit distribution on the plate interface is derived from Eqs. (10) and (18), we can compute the surface displacement rate field $u_{i}(i=x, y, z)$ using the following equation:

$$
u_{i}(\boldsymbol{x}, t)=\int_{S} U_{i}(\boldsymbol{x} ; \boldsymbol{\xi}) \Delta w_{s}(\boldsymbol{\xi}, t) d S(\boldsymbol{\xi}) .
$$

Here, $U_{i}$ denotes the response function representing the $i$ component of the displacement at $\boldsymbol{x}$ on the ground because of a unit fault slip at $\boldsymbol{\xi}$ on the plate interface. In this study, we use the analytical solution for an elastic half-space derived by Okada (1992) for $U_{i}$.

\subsection{Inversion analysis}

To estimate the slip deficit rate distribution on the plate interface from the synthetic surface displacement data, we used the inversion technique developed by Yabuki and Matsu'ura (1992). Although their method is for the coseismic crustal deformation and the slip distribution due to an earthquake, it can be applied to the interseismic crustal deformation and the slip deficit distribution (Yoshioka et al., 1993). In this section, we briefly describe the inversion method used in this study.

Letting the interseismic displacement rate at each station $\boldsymbol{d}$, parameter of the slip deficit rate distribution $\boldsymbol{a}$, and design matrix $\boldsymbol{H}$, the observation equation is constructed as follows:

$$
\boldsymbol{d}=\boldsymbol{H a}+\boldsymbol{e}
$$

Assuming that the observation error $\boldsymbol{e}$ follows the normal distribution with the variance-covariance matrix $\boldsymbol{E}$, the observation equation is written in the form of the probability density function as:

$$
\begin{aligned}
p\left(\boldsymbol{d} \mid \boldsymbol{a} ; \sigma^{2}\right)= & \left(2 \pi \sigma^{2}\right)^{-N / 2}\|\boldsymbol{E}\|^{-1 / 2} \\
& \times \exp \left[-\frac{1}{2 \sigma^{2}}(\boldsymbol{d}-\boldsymbol{H} \boldsymbol{a})^{T} \boldsymbol{E}^{-1}(\boldsymbol{d}-\boldsymbol{H a})\right],
\end{aligned}
$$

where $\sigma^{2}$ represents a hyperparameter representing the error level. In addition, the prior information on the roughness of the slip deficit rate distribution is also written in the form of a probability density function:

$$
p\left(\boldsymbol{a} ; \rho^{2}\right)=\left(2 \pi \rho^{2}\right)^{-P / 2}\left\|\boldsymbol{\Lambda}_{P}\right\|^{1 / 2} \exp \left[-\frac{1}{2 \rho^{2}} \boldsymbol{a}^{T} \boldsymbol{G} \boldsymbol{a}\right],
$$

where $\rho^{2}$ represents a hyperparameter representing the weight of the prior information, $P$ is the rank of the matrix $G,\left\|\Lambda_{P}\right\|$ represents the absolute value of the product of the non-zero eigenvalues of $\boldsymbol{G}$.
In this study, the slip deficit rate distribution on the plate interface is composed as the superposition of equallyspaced B-spline functions (Cox, 1972; de Boor, 1972). Therefore, the design matrix $\boldsymbol{H}$ in Eq. (21) consists of the elastic response and the B-spline function. On the other hand, the roughness of the slip deficit rate distribution is measured by its second-order derivatives, so that the matrix $\boldsymbol{G}$ consists of the second-order derivatives of B-spline functions.

Finally, the ratio of hyperparameters $\sigma^{2}$ and $\rho^{2}$ are determined by minimizing the Bayesian information criterion (ABIC) proposed by Akaike et al. (1980),

$$
\mathrm{ABIC}=-2 \log \int p\left(\boldsymbol{d} \mid \boldsymbol{a} ; \sigma^{2}\right) p\left(\boldsymbol{a} ; \rho^{2}\right) d \boldsymbol{a},
$$

generating the best estimation of the parameter $\boldsymbol{a}$.

\section{Model Setup and Results}

\subsection{Common conditions and terminology}

We present several numerical examples in this section. The following are the common conditions for all these cases. Lamé's constants representing the elasticity of the crust are $\lambda=40 \mathrm{GPa}$ and $\mu=40 \mathrm{GPa}$. The plate subduction rate is $5 \mathrm{~cm} \mathrm{y}^{-1}$. Random errors following the normal distribution with zero mean are added to the synthesized surface displacement data. The amount of standard deviation for the vertical component is twice that for the horizontal component.

For the discussion, we define the term "moment deficit rate" or MDR, which means the moment of the slip deficit rate on the plate interface. After this section, we often use three species of MDR: (a) "Asperity MDR" is the product of rigidity, asperity area, and the steady plate convergence rate. It corresponds to the seismic moment accumulation rate by the true coupled area. (b) "Simulated MDR" is the moment of the simulated slip deficit rate distribution on the plate interface. (c) "Estimated MDR" is the moment of the slip deficit rate distribution estimated by the geodetic data inversion analysis.

\subsection{Case 1: Isolated circular asperity}

As a basic case, we assume a single circular asperity with a diameter of $20 \mathrm{~km}$ isolated at the center of the model plate interface. The model plate interface is $60 \mathrm{~km}$ wide and long. Figure 1 shows the assumed location of the model plate interface and the surface displacement rate observation points. Figure 2 shows the assumed asperity distribution on the model plate interface. The asperity MDR in Fig. 2 is $6.32 \times 10^{17} \mathrm{~N} \mathrm{~m} \mathrm{y}^{-1}$.

Figure 3(a) shows the simulated slip deficit rate distribution for the circular asperity in Fig. 2. The slip deficit clearly appears not only in the asperity region but also around the asperity. The simulated MDR in Fig. 3(a) is $2.44 \times 10^{18} \mathrm{~N} \mathrm{~m} \mathrm{y}^{-1}$. Figure 3(b) shows the surface displacement field calculated from the slip deficit distribution shown in Fig. 3(a).

We apply the inversion analysis to the synthesized data (Fig. 3(b)). Figure 4(a) shows the estimated slip deficit distribution without noise. Compared with the true distribution, the estimated slip deficit distribution is wider, and its weighted center is slightly shifted. The estimated MDR is 


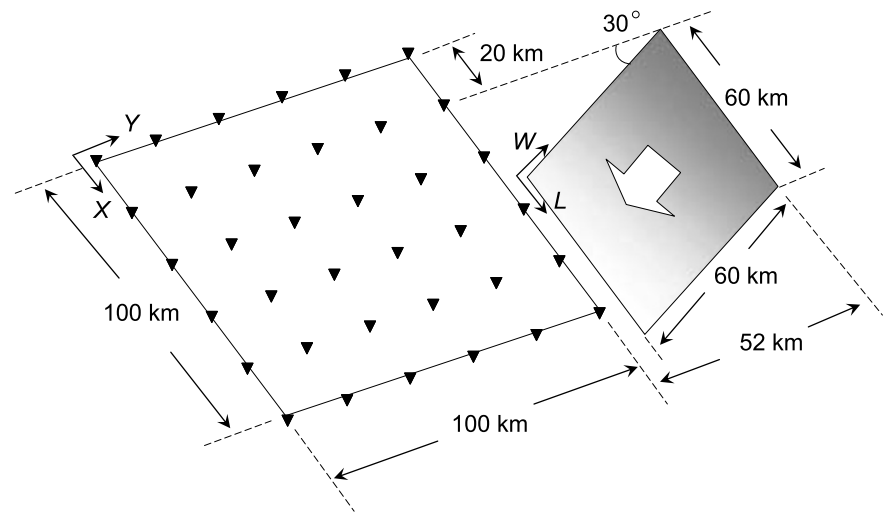

Fig. 1. The assumed location of the plate interface and surface displacement observation points. Black triangles indicate the observation points. The white arrow on the plate interface indicates the direction of plate subduction. In this study, it is parallel and opposite to the $W$ axis.

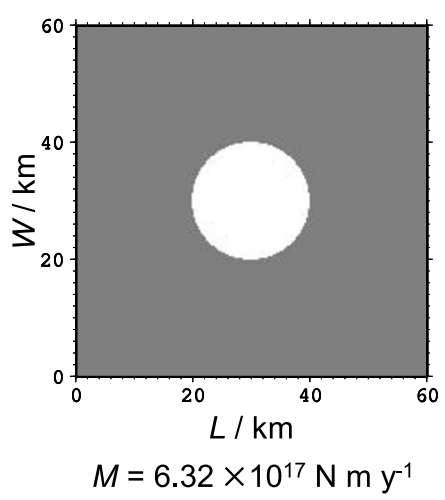

Fig. 2. Asperity distribution on the plate interface. The white area corresponds to the asperity. The gray area corresponds to the non-asperity region. The vertical axis is directed upward along the fault plane. $M$ is the MDR predicted from the asperity area and the plate convergence rate.

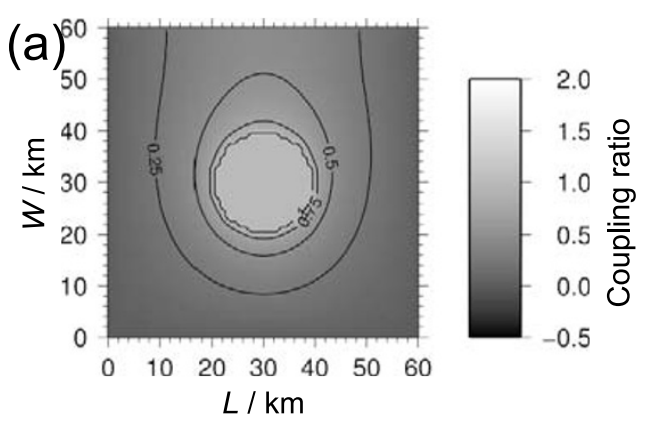

$M=2.44 \times 10^{18} \mathrm{~N} \mathrm{~m} \mathrm{y}^{-1}$

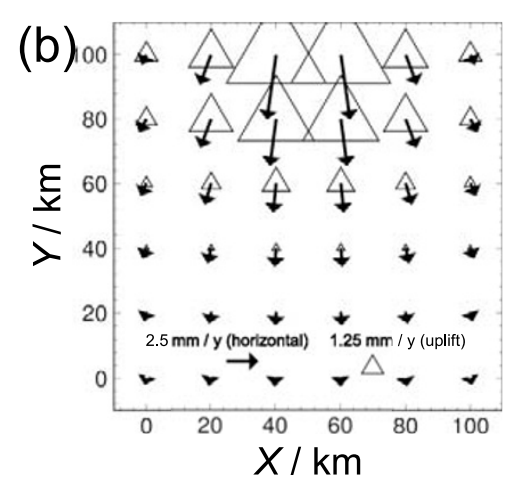

Fig. 3. (a) Calculated slip deficit rate distribution normalized by the steady plate subduction rate on the plate interface. The contour interval is 0.25 . $M$ is the simulated MDR. (b) Surface displacement rate synthesized from the slip deficit distribution rate on the plate interface. Arrows show the direction and size of the horizontal components, while triangles show the size of the vertical components. All of the vertical components are positive (uplift) in this result.

$2.71 \times 10^{18} \mathrm{~N} \mathrm{~m} \mathrm{y}^{-1}$, an $11 \%$ overestimate of the simulated MDR. Figure 4(b) shows the estimated slip deficit distribution with small random errors. A standard deviation of $2 \mathrm{~mm} \mathrm{y}^{-1}$ for the horizontal component, and $4 \mathrm{~mm} \mathrm{y}^{-1}$ for the vertical component, is assumed. The estimated MDR is $2.65 \times 10^{18} \mathrm{~N} \mathrm{~m} \mathrm{y}^{-1}$ in this case. Figure 4(c) shows the estimated slip deficit distribution with random errors whose standard deviations are twice as large as those for the case in Fig. 4(b). For this estimated slip deficit distribution, the estimated MDR is $2.60 \times 10^{18} \mathrm{~N} \mathrm{~m} \mathrm{y}^{-1}$. From these inversion results, we conclude that the estimated MDR is not very sensitive to the amount of random error.

In Fig. 4, it should be noted that the spatial distribution of the slip deficit for the error-free inversion result is deformed, while that with some error is almost circular. This implies that the realistic spatial distribution of the slip deficit rate cannot be detected; nevertheless, the observation data has enough accuracy if the physical model is 

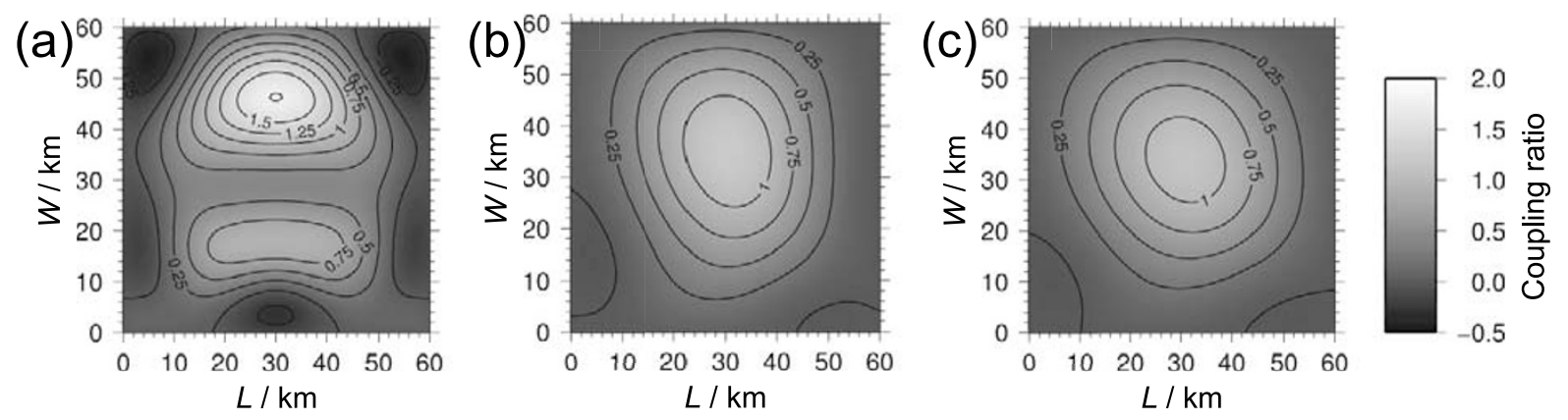

$M=2.71 \times 10^{18} \mathrm{~N} \mathrm{~m} \mathrm{y}^{-1}$

$$
M=2.65 \times 10^{18} \mathrm{~N} \mathrm{~m} \mathrm{y}^{-1}
$$

$M=2.60 \times 10^{18} \mathrm{~N} \mathrm{~m} \mathrm{y}^{-1}$

Fig. 4. The slip deficit rate distribution normalized by the steady plate subduction rate on the plate interface estimated from the synthetic surface displacement rate, (a) without noise, (b) with random errors, and (c) with larger random errors. The contour interval is $0.25 . M$ is the estimated MDR.

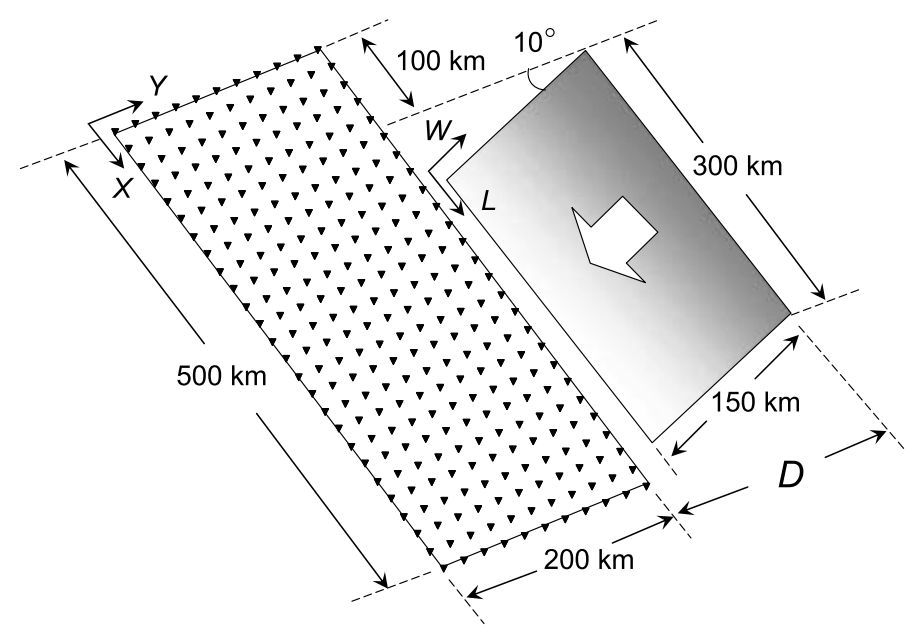

Fig. 5. The assumed location of the plate interface and the surface displacement observation points. Black triangles indicate the observation points. The white arrow on the plate interface indicates the direction of plate subduction. In this study, it is parallel and opposite to the $W$ axis. $D$ is the distance between the observation network and the trench axis.

not accurate. As an example of a similar problem, Yagi and Fukahata (2008) pointed out the importance of a nondiagonal component of the variance-covariance matrix in the maximum likelihood method. However, it is now difficult to retrieve the full variance-covariance matrix, so that the variance-covariance matrix is often assumed as a diagonal matrix for simplicity.

\subsection{Case 2: Multiple asperities}

In this section, we evaluate the relation between the seismic moment, which is calculated as the product of the asperity area and the plate convergence rate, and that estimated from geodetic data inversion. For this purpose, we conduct a series of forward calculations of surface displacement rates from randomly-distributed asperities, and inversion analysis to estimate the slip deficit rate distribution. In each case, the forward calculation and the inversion analysis are conducted as follows: (i) $\alpha$ is defined as the ratio of the asperity area to the source region where asperities are allowed to exist. The asperity distribution is generated by using random numbers so that the whole asperity area becomes $\alpha \times$ (area of the source region). (ii) The slip deficit distribution rate is calculated based on the generated asperity distribution. (iii) The surface displacement rate is calculated based on the slip deficit rate distribution on the plate interface. (iv) Random errors following a normal distribution with a standard deviation of $2 \mathrm{~mm} \mathrm{y}^{-1}$ for the horizontal components, and $4 \mathrm{~mm} \mathrm{y}^{-1} \mathrm{~mm}$ for the vertical components, are added to the synthesized surface displacement data. (v) A geodetic data inversion method is applied to the synthetic data and the slip deficit rate distribution on the plate interface is estimated.

The asperity distributions are made by generating circles with a diameter of $30 \mathrm{~km}$ in the source region that allow overlaps. Figure 5 shows the location of the model plate interface and the surface displacement observation points. Figure 6(a) shows the source region on the model plate interface. Figures 6(b) and (c) show examples of the asperity distribution for $\alpha=0.1$ and $\alpha=0.5$, respectively.

We conducted 100 forward simulations, as well as an inversion analysis for 10 cases with $\alpha=$ $0.1,0.2,0.3, \ldots, 1.0$; hence, a total of 1000 cases. In the case of $\alpha=1.0$, only one asperity distribution exists, but random errors for the surface displacement data were generated 100 times.

Figure 7 shows the relation between the asperity MDR and the estimated MDR. We observed that the smaller the asperity area, the larger is the overestimation of the MDR. Another important conclusion from Fig. 7 is that no 

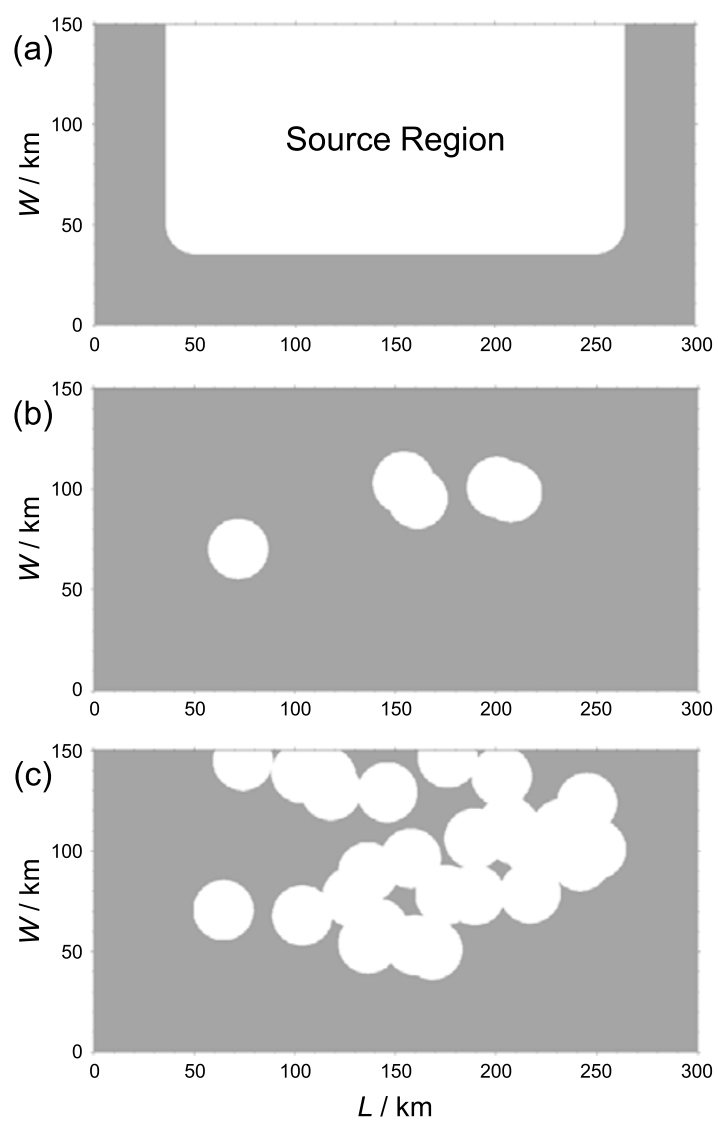

Fig. 6. (a) The source region in which an asperity is allowed to exist on the plate interface. Circular asperities with a diameter of $30 \mathrm{~km}$ are allowed to exist in the white area. (b) An example of the asperity distribution for $\alpha=0.1$ and (c) $\alpha=0.5$.

detection of slip deficit from the geodetic inversion implies virtually no asperity.

Seismic waves attenuate rapidly with distance from the seismic source. Since earthquakes are expected to occur at the asperity, it is important for the prediction of strong ground motion resulting from these earthquakes to determine the distance between the observation site and the asperity. Therefore, we checked the relation between the weighted center of the assumed asperity distribution and that of the slip deficit distribution. The weighted center $\boldsymbol{x}_{w}$ is defined as:

$$
\boldsymbol{x}_{w}=\int_{S} \rho(\boldsymbol{x}) \boldsymbol{x} d S(\boldsymbol{x}) / \int_{S} \rho(\boldsymbol{x}) d S(\boldsymbol{x}),
$$

where $\rho(\boldsymbol{x})$ is the distribution of the asperity or the slip deficit rate. The integral is applied over the whole plate interface. Although the weighted center is given in a vector form, we focus only on its down-dip component ( $W$ component) because the distance between the observation site and the asperity is considered here. Figure 8 shows the relation between the weighted center of the assumed asperity and that of the slip deficit distribution for the case of $D=100 \mathrm{~km}$ (see Fig. 5). Larger values indicate a greater distance from the observation network. According to Fig. 8(b), distances between asperities at less than $W=80 \mathrm{~km}$ and the observation network are overestimated, and vice versa. In this case, it is likely that the geodetically-

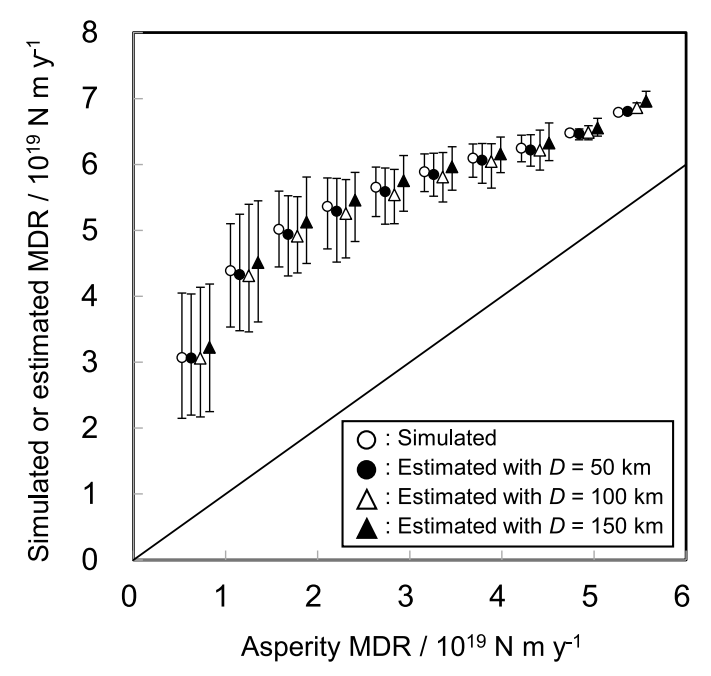

Fig. 7. The relation between the asperity MDR and the simulated and estimated MDR. Error bars denote the range of the MDR. In each group, the symbols are plotted separated for clarity although they have the same value of the asperity MDR. The straight line is proportional to a slope of 1 .

estimated slip deficit location is shifted from the asperities toward the network.

In Fig. 8(a), we can see that the weighted center of the theoretical slip deficit rate generally does not agree with that of the assumed asperity. Conversely, Fig. 8(c) shows that the weighted center of the slip deficit can be estimated approximately by geodetic data inversion. Therefore, the geodetic data inversion shows the correct slip deficit distribution while the asperity distribution may not be estimated correctly. We also compared the weighted center of the estimated slip deficit and the assumed asperities for a different distance $D$ between the observation network and the trench axis. According to the results shown in Fig. 9, the difference between the weighted center of the true asperity distribution, and that of estimated slip deficit, does not have a strong dependence on $D$. This conclusion holds even if the observation network is located above the plate interface, as is demonstrated in the case of $D=50 \mathrm{~km}$.

\subsection{Case 3: Application to real situations}

As an actual case, we applied the analyses described above to the seismogenic regions off the coasts of Tohoku and Nankai, Japan. The Pacific plate off the Tohoku district is subducting under the North American plate. Many large interplate earthquakes have occurred on the subducting plate interface. Most of these earthquakes had a magnitude of 7-8 and a recurrence period of a few decades or centuries; however, on March 11, 2011 a $M_{\mathrm{w}} 9.0$ great earthquake occurred off the Pacific coast of Tohoku. Conversely, the Philippine Sea plate is subducting under the Eurasian plate from the Nankai Trough along the Nankai district. Unlike the region off Tohoku, few small earthquakes occur in this area but many great earthquakes over $M_{\mathrm{w}} 8$ have occurred in the area with a recurrence period of 100-150 years.

3.4.1 The seismogenic region off Tohoku Figure 10 shows the location of the Tohoku district and the off Tohoku seismogenic region. The estimated slip deficit rate 

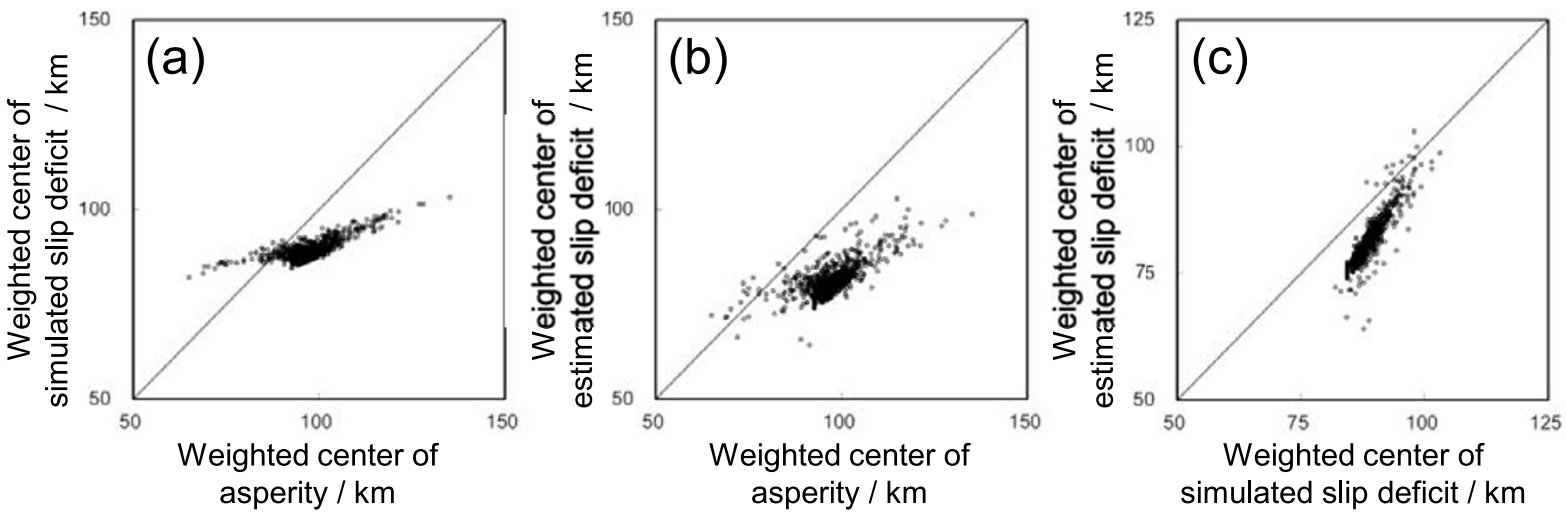

Fig. 8. (a) The relation between the weighted center of assumed asperity and that of the calculated slip deficit rate. (b) The relation between the weighted center of assumed asperity and that of the slip deficit rate estimated from the synthetic surface displacement data. (c) The relation between the weighted center of estimated slip deficit distribution and the assumed slip deficit distribution. These figures are for $D=100 \mathrm{~km}$. The straight line is proportional to a slope of 1 .

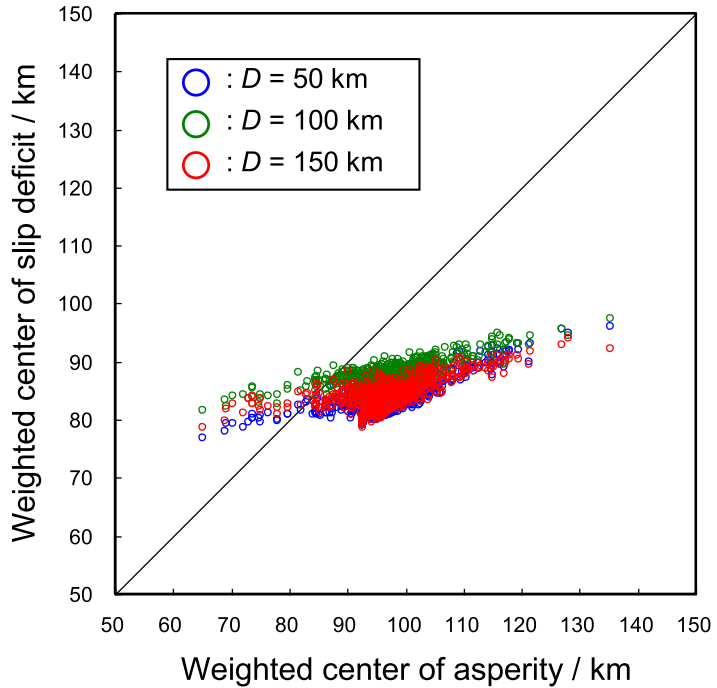

Fig. 9. The variation of the weighted center of the estimated slip deficit at a distance $D$ between the trench axis and the observation network. The straight line is proportional to a slope of 1 .

distribution and the observed and reproduced velocity are also shown in the figure. In this section, an inversion analysis is conducted using the rate of change of the baseline length, which is defined by the edges of the Delaunay triangles connecting the GPS stations. Although the Delaunay triangulation does not lead to linearly-independent baseline rates, we regarded them as a linearly-independent data set for simplicity. It may not significantly affect the conclusion since it is merely a practical problem. The slip deficit rate north of the broken line in Fig. 10 is used for the calculation of the MDR. The assumed seismic fault on the plate interface is $510-\mathrm{km}$ long and $210-\mathrm{km}$ wide. The strike and dip angles are $195^{\circ}$ and $13^{\circ}$, respectively. The plate convergence rate is assumed to be $8 \mathrm{~cm} \mathrm{y}^{-1}$. The MDR was estimated at $1.8 \times 10^{20} \mathrm{~N} \mathrm{~m} \mathrm{y}^{-1}$. The result shown in Fig. 10 is consistent with previous studies (e.g., Nishimura et al., 2004; Hashimoto et al., 2009). We also conducted a series of forward simulations of the surface displacement rate and inversion analysis to estimate the slip deficit rate distri-

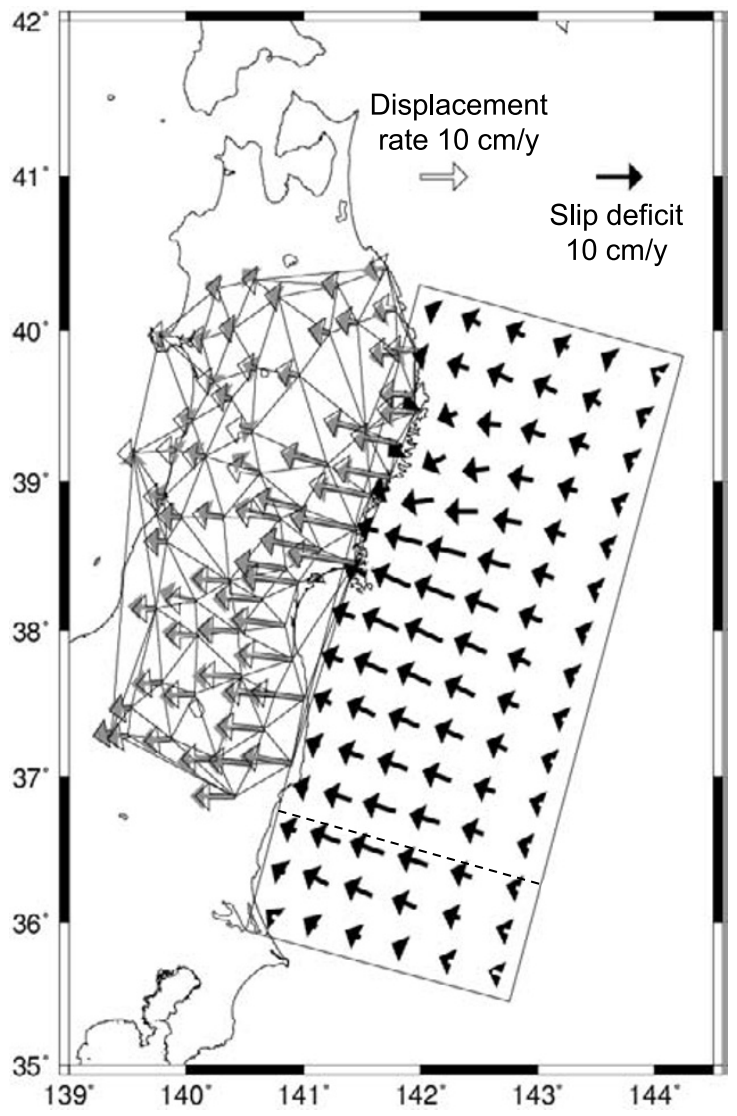

Fig. 10. The location of the Tohoku district of Japan and the seismogenic region off Tohoku. The rectangle indicates the seismic fault model used for the forward simulation and inversion analysis. Black arrows indicate the estimated slip deficit rate distribution by geodetic data inversion. Gray and white arrows indicate the horizontal velocity vectors at GPS stations relative to a reference point for the interseismic calm period 1996-2000 and those reproduced from the estimated slip deficit rate distribution. Solid lines connecting the GPS stations define the Delaunay triangles.

bution as in Subsection 3.3. Figure 11 shows the relation between the asperity MDR and the estimated MDR. The MDR estimated from the real geodetic data lies at the middle of the curve in Fig. 11, representing a moderate coupling 


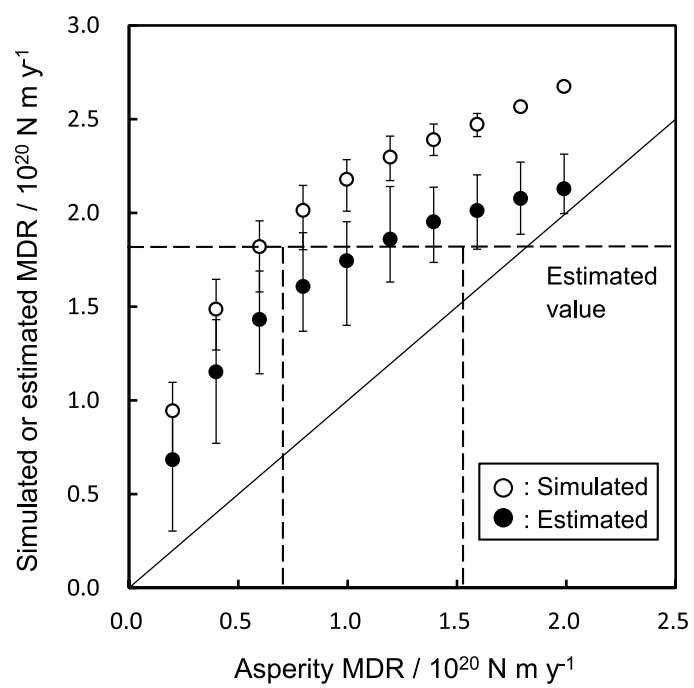

Fig. 11. The relation between the asperity MDR and the simulated and estimated MDR for the seismogenic region off Tohoku. Error bars denote the range of the MDR by asperity distribution. The straight line is proportional to a slope of 1 .

ratio. According to Fig. 11, the asperity MDR may be approximately $0.7-1.5 \times 10^{20} \mathrm{~N} \mathrm{~m} \mathrm{y}^{-1}$. According to Ito et al. (2011), a seismic moment of $4.1 \times 10^{22} \mathrm{~N} \mathrm{~m}\left(M_{\mathrm{w}}=9.0\right)$ was released in the 2011 earthquake off the Pacific coast of Tohoku. It is comparable with $270-570$ years accumulation of the regular seismic moment corresponding to the asperity area. However, the accumulated seismic moment is released not only by the $M_{\mathrm{w}} 9$ earthquake but also by earthquakes under $M_{\mathrm{w}} 9$ and by aseismic slips in this region (see Kawasaki et al., 2001). Including these effects, the recurrence period of the $M_{\mathrm{w}} 9$ earthquake is longer than stated above. If the MDR of this region and the history of past $M_{\mathrm{w}} 9$ earthquakes had been revealed, the potential for the $M_{\mathrm{w}} 9$ earthquake in this region, such as the 2011 earthquake, might have been predicted.

The result of the inversion analysis may change depending on the regularizing function, the boundary condition of the slip deficit, manipulation of the viscoelastic effect, and so on. However, the effect of a choice of the inversion method is not important in this study, since we discuss the relation between the mechanical coupling of the plate interface and the slip deficit estimated in a kinematic manner. In this study, we used the method developed by Yabuki and Matsu'ura (1992) as one of the most popular inversion methods.

3.4.2 The Nankai seismogenic region Figure 12 shows the location of the Nankai seismogenic region. As in the case of the Tohoku district, the estimated slip deficit rate distribution and the observed and reproduced velocities are shown (Fig. 12). The solid lines connecting the GPS stations define the edges of the Delaunay triangles. The slip deficit rate on the west of the broken line in Fig. 12 is used for the calculation of the MDR. The assumed seismic fault on the plate interface is $350-\mathrm{km}$ long and $150-\mathrm{km}$ wide. The strike and dip angles are $248^{\circ}$ and $14^{\circ}$, respectively. The plate convergence rate is assumed to be $6 \mathrm{~cm} \mathrm{y}^{-1}$. Using the real geodetic data, the estimates for the slip deficit

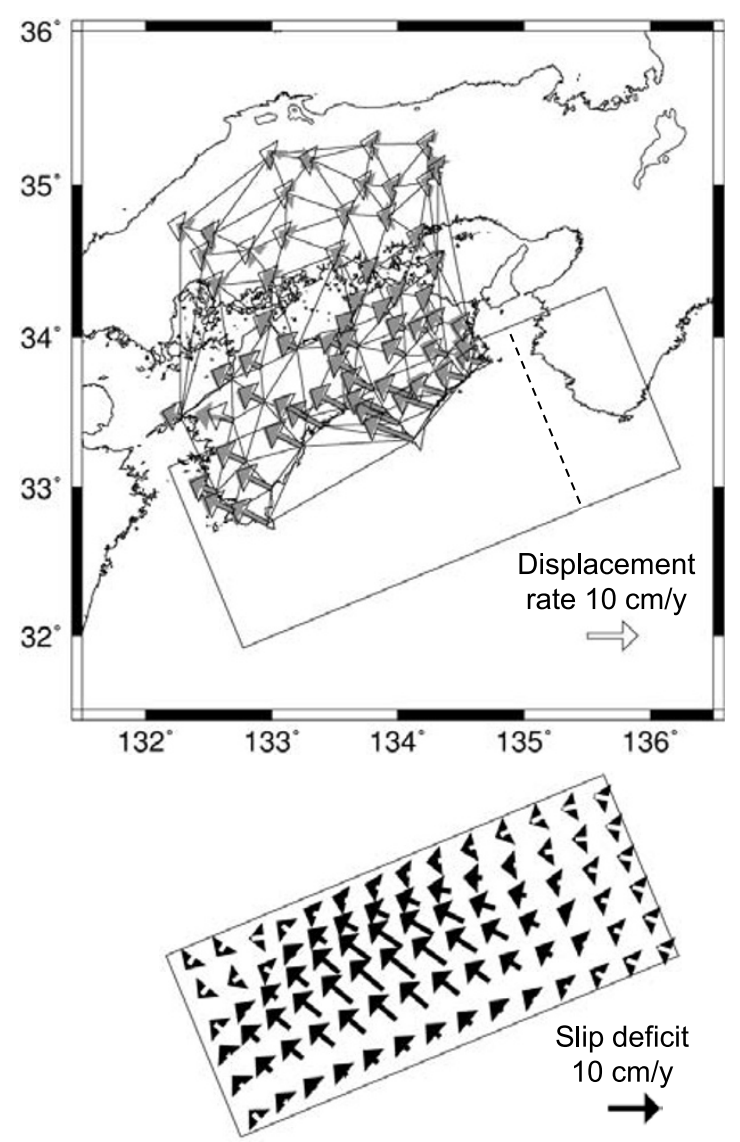

Fig. 12. The location of the Nankai seismogenic region. The rectangle indicates the seismic fault model used for the forward simulation and inversion analysis. Black arrows shown in the lower rectangle indicate the estimated slip deficit rate distribution by geodetic data inversion. Gray and white arrows indicate the horizontal velocity vectors at the GPS stations relative to a reference point for the interseismic calm period of 1996-2000 and those reproduced from the estimated slip deficit rate distribution. Solid lines connecting the GPS stations define the Delaunay triangles.

rate distribution are shown in Fig. 12. The estimated MDR is $6.7 \times 10^{19} \mathrm{~N} \mathrm{~m} \mathrm{y}^{-1}$ in this case. The results shown in Fig. 12 are consistent with previous studies (e.g., Miyazaki and Heki, 2001). We conducted a series of forward simulation and inversion analysis, similar to those for the region off Tohoku. The results are shown in Fig. 13. The MDR estimated from the real geodetic data lies almost on top of the curve in Fig. 13. The results indicate that the asperity MDR may be greater than approximately $3.6 \times 10^{19} \mathrm{~N} \mathrm{~m} \mathrm{y}^{-1}$. In this case, the degree of interplate coupling-whether it is moderate, or reaches almost complete locking-cannot be determined because the curve in Fig. 13 has a small derivative near the MDR estimated from the real geodetic data. The estimated MDR is systematically underestimated for the Nankai earthquake in this study because the seismic moment calculation area occupies about $80 \%$ of the Nankai seismogenic region. Therefore, the true MDR is slightly greater than the estimated MDR. For example, if the real MDR is $5 \times 10^{19} \mathrm{~N} \mathrm{~m} \mathrm{y}^{-1}$, an $M_{\mathrm{w}} 8.4$ earthquake will develop over 100 years. This estimation is consistent with moment magnitudes of past Nankai earthquakes. The agreement between the moment magnitudes of past Nankai 


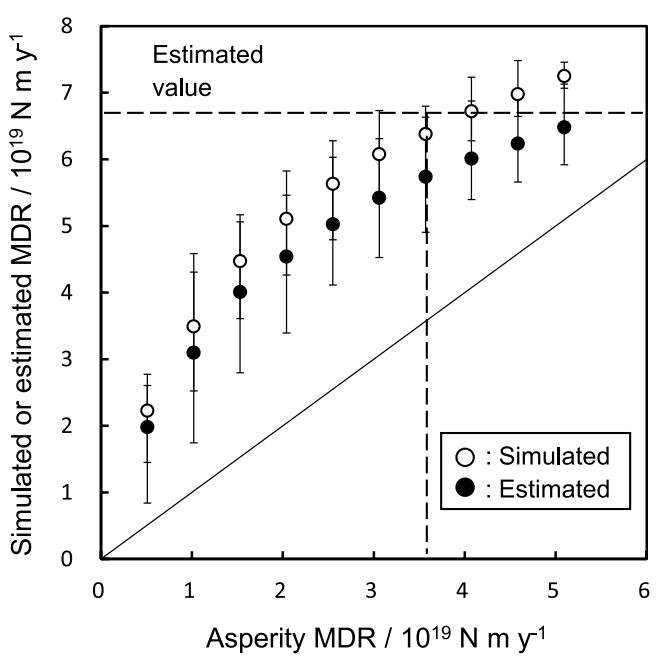

Fig. 13. The relation between the asperity MDR and the simulated and estimated MDR for the Nankai seismogenic region. Error bars denote the range of the MDR by asperity distribution. The straight line is proportional to a slope of 1 .

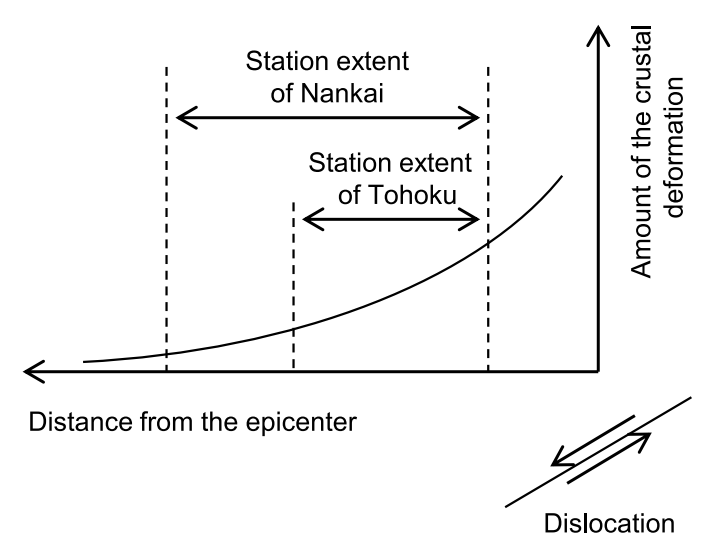

Fig. 14. Schematic diagram of the plate subduction zone and the distribution of interseismic displacement rate due to the interplate locking.

earthquakes and those estimated from the geodetic data corresponds with our knowledge that very few smaller earthquakes occur in this region around the time of the $M_{\mathrm{w}} 8$ Nankai earthquakes.

The estimated MDR is evidently smaller than simulated MDR in Figs. 11 and 13, while they are close enough in Fig. 7. That is because the change rate of baseline length is used as the observation data in Subsection 3.4, while the displacement rate is used as the observation data in Subsection 3.3. The displacement pattern due to the interplate coupling is as shown in Fig. 14. When using the change rate of baseline length as the observation data, the absolute level of the displacement rate is not taken into account, causing the underestimation of the MDR. The narrower is the observation network, the more notably this effect appears. So that the difference between simulated and estimated MDR is smaller for the Nankai seismogenic region than for the off Tohoku region, since the observation network of the Nankai region covers a broader area up to the convergence of the displacement field.

\section{Discussion}

First, we compare the asperity MDR, simulated MDR, and estimated MDR. According to the results in Subsection 3.2, the simulated MDR and the estimated MDR are relatively close, while the asperity MDR is evidently smaller. The result in Subsection 3.3 also shows the same tendency, and does not depend on the distance between the observation network and the plate interface. The reason for the simulated MDR being greater than the asperity MDR is that the slip deficit, because of the plate motion, is generated not only in the asperity but also around the asperity. The closeness between the simulated and estimated MDR means that the overall amount of slip deficit can be estimated correctly, while the difference between the asperity MDR and the estimated MDR indicates that the true amount of interplate coupling is overestimated by the geodetic data inversion. Asperity is considered to have a frictional property of the slip-weakening type, while non-asperity areas are characterized by slip-strengthening friction. According to the rate and state dependent friction law, their behavior corresponds to the negative and positive value of the parameter $a-b$ (see Ruina, 1983), respectively. Assuming that high-speed fault rupture occurs only in the asperity and will stop when the fault rupture reaches the surrounding area, the difference between the asperity MDR and the estimated MDR indicates that the seismic moment of the earthquake generated at that asperity is overestimated by the geodetic data inversion. The remaining seismic moment would probably be released by a slow slip process, with a significant portion of this slip occurring during postseismic, or interseismic, periods. This assumption stands for an extreme case, fault rupture may also occur in a part of non-asperity in fact; even so, we can say that geodetic inversion of interseismic crustal deformation tends to overestimate the seismic moment of future earthquakes.

As discussed in Subsection 3.4, in general, the MDR may be overestimated from real geodetic data. In the case of largely-estimated MDR, we cannot determine whether the asperity area is moderate, or almost complete locking. The Nankai district of Japan corresponds to this case. In contrast, seismic moment curves like those in Figs. 7, 11, and 13 , suggest that asperity does not exist on the plate interface when no slip deficit is detected by geodetic data inversion. For example, GPS observations have revealed an oceanward motion of the coastal area of the Ryukyu Trench, off southern Kyushu Island, Japan (Imanishi et al., 1996; Kotake et al., 1998). Figure 15 shows the location of the Ryukyu Trench and some tectonic settings. The orientation of this motion is opposite to the Philippine Sea plate subduction. Such an oceanward motion cannot be explained by interplate coupling between the subducting Philippine Sea plate and the overriding Eurasian plate. Thus, this region is a potential case of minimal interplate coupling. For Nankai, Tohoku, and Ryukyu districts, the MDRs are estimated as large, moderate, and small values, respectively, indicating the possibility of maximum asperity in those areas. Each relation corresponds to the degree of interplate coupling; however, this is not clear in the Nankai seismogenic region. These results reflect the spatial variety of interplate coupling among each subduction zone. When estimating 


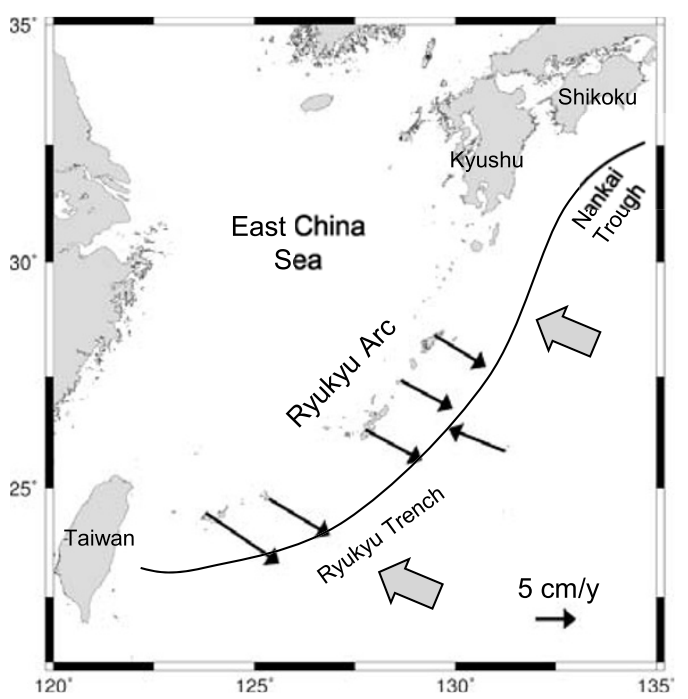

Fig. 15. The location of the coastal area of the Ryukyu Trench. The black arrows indicate the direction of displacement during 2006 observed by GPS. The gray arrows indicate the relative motion of the Philippine Sea plate with respect to the Eurasian plate. The relative plate motion is calculated based on Sella et al. (2002).

the seismic hazard of the plate interface, the dispersion of the seismic moment often becomes a problem. Applying a series of forward simulation and inversion analysis to the objective seismogenic region, the dispersion of seismic moment can be estimated quantitatively. In addition, the degree of interplate coupling may be revealed by comparing the MDR estimated from real geodetic data with the numerical analysis described in this study.

As shown in Figs. 10 and 12, the slip deficit rate is estimated to be small near the trench. In these parts, the solution of the slip deficit rate is strongly influenced by the boundary condition. However, the geodetic data inversion method has a poor resolution near the trench area because of the large distance from the observation network. Therefore, interplate coupling occurring offshore cannot be detected accurately, even though such motion can generate strong and destructive tsunamis.

\section{Conclusion}

We evaluated the physical state of the plate interface estimated from the interseismic crustal deformation rate, combining the forward simulation of crustal deformation because of plate subduction and geodetic data inversion analysis. Significant results obtained here are as follows: (1) the slip deficit distribution on the model plate interface can be estimated with some accuracy although the spatial resolution is limited. The estimated moment deficit rate (MDR) is larger than that expected from the asperity distribution. This could lead to an overestimation of the seismic moment of a future earthquake. The tendency to overestimate the MDR is strong when the asperities occupy a small area on the plate interface. In the opposite sense, if no slip deficit is detected by geodetic data inversion, there is no asperity on the plate interface. (2) The weighted center of the slip deficit often does not agree with that of the asperity distribution. Since seismic waves attenuate with distance from the seismic source, the difference between the weighted center of the asperity distribution and that of the estimated slip deficit distribution may cause an inaccuracy in predicting strong ground motion by a future earthquake. (3) A variation in the interplate coupling among the subduction zones off Tohoku, Nankai, and Ryukyu were revealed. We conducted a series of forward simulation and inversion analyses, and found the relation between the estimated MDR and the true asperity area for each subduction zone. Comparing this with the MDR estimated from real geodetic data, moderate and almost complete locking of the plate interface was confirmed for the seismogenic regions off Tohoku and Nankai, respectively.

Acknowledgments. The GPS data used in this study was provided by the Geospatial Information Authority of Japan. We acknowledge the comments by Dr. Paul Segall and one anonymous reviewer in improving the manuscript. We would like to thank Enago (www.enago.jp) for the English language review. The figures were prepared using Generic Mapping Tools (Wessel and Smith, 1995).

\section{Appendix A. Derivation of the Shear Stress Re- sponse Function}

In this appendix we give the derivation of the stress response function $H(\boldsymbol{x} ; \boldsymbol{\xi})$ used in Subsection 2.1.

Since $H(x ; \xi)$ represents the down-dip component of the stress vector, it is given as a product of the transpose of the unit vector along the subduction direction $\boldsymbol{v}^{T}(\boldsymbol{x})$, stress tensor $\boldsymbol{H}(\boldsymbol{x} ; \boldsymbol{\xi})$, and unit normal vector of the plate interface $n(x)$ :

$$
H(x ; \xi)=v^{T}(x) H(x ; \xi) n(x) .
$$

Assuming the substratum is an isotropic linear elastic body, the relation between the $i j$-component of the stress tensor and the internal deformation is given as:

$$
\begin{aligned}
H_{i j}(\boldsymbol{x} ; \boldsymbol{\xi})= & \delta_{i j} \lambda \sum_{k=1}^{3} \frac{\partial U_{k}(\boldsymbol{x} ; \boldsymbol{\xi})}{\partial x_{k}} \\
& +\mu\left[\frac{\partial U_{i}(\boldsymbol{x} ; \boldsymbol{\xi})}{\partial x_{j}}+\frac{\partial U_{j}(\boldsymbol{x} ; \boldsymbol{\xi})}{\partial x_{i}}\right] \\
& (i, j=1,2,3)
\end{aligned}
$$

where $\delta_{i j}$ is Kronecker's delta, $\lambda$ and $\mu$ are Lamé's constants, and $U_{i}(\boldsymbol{x} ; \boldsymbol{\xi})$ is the $i$-component of displacement at $\boldsymbol{x}$ due to a unit fault slip at $\boldsymbol{\xi}$, respectively. The detailed expressions of $\partial U_{i}(\boldsymbol{x} ; \boldsymbol{\xi}) / \partial x_{j}$ are given in Okada (1992).

\section{References}

Akaike, H., J. M. Bernardo, M. H. DeGroot, D. V. Lindley, and A. F. M. Smith, Likelihood and the Bayes procedure, in Bayesian Statistics, 143166, University Press, Valencia, Spain, 1980.

Carlson, J. M., J. S. Langer, B. E. Shaw, and C. Tang, Intrinsic properties of a Burridge-Knopoff model of an earthquake fault, Phys. Rev. A, 44, 884-897, 1991.

Cox, M. G., The numerical evaluation of B-splines, J. Inst. Math. Appl., 10, 134-149, 1972.

de Boor, C., On calculating with B-splines, J. Approx. Theory, 6, 50-62, 1972.

Dieterich, J. H., Modeling of rock friction, 1, Experimental results and constitutive equations, J. Geophys. Res., 84, 2161-2168, 1979.

Harris, R. A. and P. Segall, Detection of a locked zone on the Parkfield, California, segment of the San Andreas Fault, J. Geophys. Res., 92, 7945-7962, 1987. 
Hashimoto, C., A. Noda, T. Sagiya, and M. Matsu'ura, Interplate seismogenic zones along the Kuril-Japan trench inferred from GPS data inversion, Nat. Geosci., 2, 141-144, 2009.

Imanishi, M., F. Kimata, N. Inamori, R. Miyajima, T. Okuda, K. Takai, K. Hirahara, and K. Kato, Horizontal displacements by GPS measurements at the Okinawa-Sakishima Islands (1994-1995), J. Seismol. Soc. Jpn., 49, 417-421, 1996.

Ito, T., S. Yoshioka, and S. Miyazaki, Interplate coupling in southwest Japan deduced from inversion analysis of GPS data, Phys. Earth Planet. Inter., 115, 17-34, 1999.

Ito, T., K. Ozawa, T. Watanabe, and T. Sagiya, Slip distribution of the 2011 off the Pacific coast of Tohoku Earthquake inferred from geodetic data, Earth Plantes Space, 63, 627-630, 2011.

Kawasaki, I., Y. Asai, and Y. Tamura, Space-time distribution of interplate moment release including slow earthquakes and the seismo-geodetic coupling in the Sanriku-oki region along the Japan trench, Tectonophysics, 330, 267-283, 2001.

Kikuchi, M., M. Nakamura, and K. Yoshikawa, Source rupture processes of the 1944 Tonankai earthquake and the 1945 Mikawa earthquake derived from low-gain seismograms, Earth Planets Space, 55, 159-172, 2003.

Kotake, Y., T. Kato, S. Miyazaki, and A. Sengoku, Relative motion of the Philippine Sea plate derived from GPS observations and tectonics of the south-western Japan, J. Seismol. Soc. Jpn., 51, 171-180, 1998.

Lay, T. and H. Kanamori, An asperity model of large earthquake sequences, in Earthquake Prediction, An International Review, edited by D. Simpson and P. Richard, 579-592, Maurice Ewing Series, 4, AGU, Washington, D.C., 1981.

Miyazaki, S. and K. Heki, Crustal velocity field of southwest Japan: Subduction and arc-arc collision, J. Geophys. Res., 106(B3), 4305-4326, 2001.

Moreno, M., M. Rosenau, and O. Oncken, 2010 Maule earthquake slip correlates with pre-seismic locking of Andean subduction zone, Nature, 467, 198-202, 2010.

Moreno, M., D. Melnic, M. Rosenau, J. Baez, J. Klotz, O. Oncken, A. Tassara, J. Chen, K. Bataille, M. Bevis, A. Socquet, J. Bolte, C. Vigny, B. Brooks, I. Ryder, V. Grund, B. Smalley, D. Carrizo, M. Bartsch, and H. Hase, Toward understanding tectonic control on the $M_{\mathrm{w}} 8.82010$ Maule Chile earthquake, Earth Planet. Sci. Lett., 321-322, 152-165,
2012.

Nagai, R., M. Kikuchi, and Y. Yamanaka, Comparative study on the source processes of recurrent large earthquakes in Sanriku-oki region: the 1968 Tokachi-oki Earthquake and the 1994 Sanriku-oki Earthquake, Zisin Second Ser., 64, 267-280, 2001 (in Japanese).

Nishimura, T., T. Hirasawa, S. Miyazaki, T. Sagiya, T. Tada, S. Miura, and $\mathrm{K}$. Tanaka, Temporal change of interplate coupling in northeastern Japan during 1995-2002 estimated from continuous GPS observations, Geophys. J. Int., 157, 901-916, 2004.

Ohta, Y., F. Kimata, and T. Sagiya, Reexamination of the interplate coupling in the Tokai region, central Japan, based on the GPS data in 19972002, Geophys. Res. Lett., 31, L24604, 2004.

Okada, Y., Internal deformation due to shear and tensile faults in a halfspace, Bull. Seismol. Soc. Am., 82, 1018-1040, 1992.

Ruina, A., Slip instability and state variable friction laws, J. Geophys. Res., 98, 9885-9907, 1983.

Sagiya, T., Interplate coupling in the Tokai District, Central Japan, deduced from continuous GPS data, Geophys. Res. Lett., 26, 2315-2318, 1999.

Savage, J. C., A dislocation model of strain accumulation and release at a subduction zone, J. Geophys. Res., 88, 4984-4996, 1983.

Sella, G. F., T. H. Dixon, and A. Mao, REVEL: A model for Recent plate velocities from space geodesy, J. Geophys. Res., 107(B4), 2081, 2002.

Yabuki, T. and M. Matsu'ura, Geodetic data inversion using a Bayesian information criterion for spatial distribution of fault slip, Geohpys. $J$. Int., 109, 363-375, 1992.

Yagi, Y. and Y. Fukahata, Importance of covariance components in inversion analyses of densely sampled observed data: An application to waveform data inversion for seismic source processes, Geophys. J. Int., 175, 215-221, 2008.

Yoshioka, S., T. Yabuki, T. Sagiya, T. Tada, and M. Matsu'ura, Interplate coupling and relative plate motion in the Tokai district, central Japan, deduced from geodetic data inversion using ABIC, Geophys. J. Int., 113, 607-621, 1993.

Wessel, P. and W. H. F. Smith, New version of the Generic Mapping Tools released, Eos Trans. AGU, 76, 329, 1995.

T. Hirai (e-mail: hirai@sharaku.nuac.nagoya-u.ac.jp) and T. Sagiya 